\title{
Correspondence
}

\section{Continuing care provision for the mentally ill}

\section{DeAR Sirs}

Profound anxieties are evident among practitioners in old age psychiatry and other psychiatric specialties that appropriate long-term care for the most severely disabled/disturbed is becoming increasingly difficult to ensure as health authorities have closed longstay beds and assumed or arranged that alternatives will be provided by 'independent', non-hospital agencies.

It may be helpful for practitioners to know of letters issued from the Department of Health and NHS Management Executive earlier this year.

24 January 1992. Stephen Dorrell wrote from the Department of Health to the Chairman of Newcastle Health Authority rejecting that Health Authority's plan to 'reprovide' services of Ponteland and Lemmington Hospitals through a collaborative scheme with Anchor Housing.

"I recognise that your proposals would have provided an improved physical environment for the elderly people concerned, and I welcome imaginative proposals for co-operation between the Health Service and the independent sector. Your proposals would, however, have involved a wholesale switch of financial responsibility from the Health Authority to the Department of Social Security. The patients concerned would therefore have effectively been discharged from NHS in-patient care not as a result of a decision by the clinician responsible for their care, but as a result of a shift of financial responsibility from the Health Authority to the Department of Social Security.

Health Authorities should not, of course, assume responsibility for people who do not have continuing health care needs. Health Authorities do, however, have - and will continue to have - a responsibility to provide or secure long-term care for those people who need it by reason of the predominance of their continuing ill-health. This point is made clear in the White Paper 'Caring for People' (paragraphs 4.20-4.21) of which I attach a copy."

7 February 1992. Andrew Foster wrote to all Regional General Managers identifying four 'must be done' issues in the implementation of 'Caring for People':

(1) clarifying and agreeing with Local Authorities continuing care arrangements

(2) agreeing and preparing for health involvement in needs based assessment
(3) ensuring the robustness of discharge arrangements

(4) clarifying the roles of GPs and primary care teams

and amplifying consideration of these in an accompanying paper.

'Continuing Care'. DHAs to establish a clear policy in relation to the NHS purchase of continuing care (continuing care beds, respite care, rehabilitation and community services), identifying and quantifying their current commitments including all those which they share with other agencies e.g. Section 64 grants and protecting those resources. As part of the 1993/94 community care plans DHAs must agree with LAs an overall strategy for the commissioning of continuing care and covering; service volumes, service types, service quality, information requirements and any joint commissioning arrangements. It is not acceptable for DHAs to take unilateral decisions to withdraw from continuing care'.

It is all too easy for practitioners to feel left out of the planning dialogue - some will find 'possession' of the information contained in these letters helpful in contributing their advice locally. I am asked by our Executive to draw attention to them through the columns of the Psychiatric Bulletin.

DAVID J. JOLLEY Chairman, Old Age Section, RCPsych

\section{NHS managers and clinical management}

DeAr Sirs

A 19-year-old schizophrenic student appealed to the managers against detention under Section 3 Mental Health Act. At the time of her appeal her mental state was beginning to improve both in terms of her self care and sociability. In addition she had stopped acting on the delusional belief that her father had stolen her face. Prior to admission she had been very angry with him for this and had tried to hire a private detective to assist her in obtaining the restoration of her features. She had not washed or changed her clothes for several weeks and on one occasion had attacked her mother with a pair of scissors.

Her improvement had been slow and the multidisciplinary team considered that she needed to remain in hospital for a few more weeks so that depot rather than oral medication could be commenced (previous failed compliance with oral medication had led to this admission). The team also wished to 